\title{
Reden wir über Geld! Aber wie? Und wozu?
}

MONIKA DOMMANN

01. Oktober 2010, Süddeutsche Zeitung, in der Rubrik »Geld« (Mühlauer/Wilhelm 2010): Der Übersetzer Ulrich Blumenbach, dessen 1545 Seiten umfassende Übersetzung von David Foster Wallaces Infinite Jest zwei Jahre zuvor in der Rubrik »Feuilleton« abgefeiert worden ist, wird interviewt. Das Gespräch beginnt mit einer Aufforderung: »Herr Blumenbach, reden wir über Geld« und einer Frage: »Haben Sie schon mal ihren Stundenlohn ausgerechnet?«

Wir erfahren dann, dass der Übersetzer drei Euro pro Seite am Kultbuch verdient hat, seinen Lebensunterhalt massgeblich mit der Übersetzung von Börsennachrichten und Unternehmensmeldungen einer Schweizer Bank bestreitet und bis heute mit Infinite Chest (inklusive Preisen und Tantiemen) nicht in der »Gewinnzone« angelangt sei: »Ich habe jetzt fünf Sechstel meiner Ausgaben während der Übersetzungszeit reingeholt«. Wir lesen zudem, dass der gestiegene Bekanntheitsgrad dazu führte, dass dem Übersetzer nun »weniger, aber dafür höherwertige Literatur« angeboten wird. Und wir werden darüber in Kenntnis gesetzt, dass »Hochliteratur« zwar besser bezahlt ist als »Genreliteratur«, jedoch viel mehr Zeit in Anspruch nimmt für die Übersetzung: »Das heißt: Ich bekomme am Ende viel weniger Geld raus« (Zitate Blumenbach). Und wir erfahren, dass der Übersetzer nicht im preisgünstigen Berlin, sondern im teuren Basel wohnt (wegen seiner Schweizer Frau) und sich nun überlegt, Schweizer zu werden. Kostenpunkt: je nach Kanton zwischen 500 und 3000 Franken.

Wie verändert sich der Blick auf Literatur, wenn sie statt im Feuilleton im Wirtschaftsteil der Zeitung behandelt wird? Was bedeutet es, wenn literarische Übersetzungen in die Welt der Wirtschaft übersetzt werden? Welche Beziehungen haben die beiden Welten zueinander, die zumindest in der Person des Übersetzers, der tagsüber Literatur und nachts Börsenkurse übersetzt, aufeinandertreffen? Welche Ursachen und Folgen hat es, dass der Übersetzer sich als Unterneh- 
mer thematisiert? Warum korreliert die Übersetzung hochwertiger Literatur mit einem vergleichsweise niedrigen Arbeitslohn? Könnten solche Fragen produktiv sein für die Geisteswissenschaften? Und was würde die Absenz solcher Fragen bedeuten? Würde sie überhaupt ins Gewicht fallen?

\section{Von der Knappheit zum Knappheitsparadigma}

Wie wir im Wörterbuch der Gebrüder Grimm nachlesen können, ist das Wort »knapp« erst im Neuhochdeutschen gebräuchlich und bezeichnet enge, zu genau bemessene und beschränkte Kleidungsstücke und Räume, im übertragenen Sinn auch Geld, Zeit, das Auskommen, die Lebensverhältnisse und einen konzisen, präzisen Sprachstil. »Knapp« wird zudem als ein starkes »kaum«, als »genau bemessen « und für flinke und gewandte Personen verwendet (Grimm 1873: Bd. 5, Sp. 1338 bis 1348). Der entsprechende englischsprachige Begriff »scarcity« leitet sich von »scarce« ab (aus dem anglofranzösischen »eschars/escars«). Er wird zur Bezeichnung von Beschränkungen in Quantität, Größe und Menge verwendet. Erwähnt werden Winde, Wasser, Esswaren und gar Zeitperioden (vgl. http://www.merriam-webster.com/dictionary).

In den Wirtschaftswissenschaften ist der Begriff der »Knappheit« beziehungsweise »scarcity« zum Schlüsselbegriff geworden, seit der britische Ökonom Lionel Robbins das Handeln mit knappen Mitteln im Jahr 1932 zur Kernfrage der ökonomischen Wissenschaft erklärt hat. Knappheit meint hier jedoch nicht wie in der Alltagssprache materielle Beschränkungen als Zustand, sondern bezeichnet ein universelles Denkprinzip: »Human behaviour on disposing of scarce means«, darum gehe es in der ökonomischen Wissenschaft (Robbins1952: 15). Neben materiellen Dingen geht es auch um die eigene Zeit und die Dienstleistungen anderer. Ökonomie ist für Robins nicht die Wissenschaft des materiellen Wohlstandes, wie etwa noch beim Kameralisten und Bergwerksleiter Johann Heinrich Gottlob von Justi zur Mitte des 18. Jahrhunderts, der die Kameralwissenschaft als jene Wissenschaft bezeichnete, welche Grundsätze und Regeln beschreibt, nach denen "gemeinschaftliche Glückseligkeit» bewirkt wird (Justi 1766: 3). Oder beim politischen schottischen Philosophen und Nationalökonomen Adam Smith, der sich für die Ursachen der Verbesserung der produktiven Kräfte der Arbeit durch Arbeitsteilung interessierte (Smith 1789).

Für Lionel Robbins ist Ökonomie die Wissenschaft, die sich mit der Verwendung knapper Mittel und den damit einhergehenden Wahlkonflikten oder Wahlzwängen beschäftigt. Knappheit wird zum analytischen Konzept. Jegliches menschliche Tun und Handeln kann im Rahmen von Knappheitsbeziehungen zu einem potentiellen Objekt ökonomischer Wissenschaften werden. Dieser definitorische Akt ebnete das Terrain für eine Expansion des ökonomischen Denkens in neue Wissensgebiete. Gemäß Robbins kann selbst die literaturwissenschaftliche Frage »Did Bacon Write Shakespeare?« zur ökonomischen Frage werden unter der Vorannahme der Existenz zeitlich unbeschränkter Copyrights. Und zwar nicht weil mit Copyrights materielle Einkünfte in Verbindung stünden, son- 
sondern weil Theaterstücke durch die Existenz von Copyrights anders verwendet und verbreitet würden als ohne Copyright. Wenn der Gebrauch von Theaterstücken von der Einwilligung und/oder der Entlohnung des Rechteeigentümers abhänge, führe dies zu einer Verknappung ihrer potentiellen Verwendung (Robbins 1932: 23). Lionel Robbins war nicht der einzige Ökonom, der das Grundproblem der Ökonomie zu Beginn der 1930er Jahre in der Beschäftigung mit Wahlakten sah. Der österreichische Ökonom Ludwig Mises formulierte im Jahr 1933 bei seinen Überlegungen zur »Umgrenzung des >Wirtschaftlichen«" (Mises 1933: 137) eine Absage an die Trennung zwischen dem ")Wirtschaftlichen >Nichtwirtschaftlichen«", die von den deutschen Ökonomen zu Beginn des 20. Jahrhunderts vertreten wurde. Für Gustav Schmoller beispielsweise war die Definition des Wirtschaftens in Ableitung aus dem griechischen Oikos (Haus) an einen sozialen Körper, d. h. die Organisation des wirtschaftlichen Zusammenlebens geknüpft gewesen (Schmoller 1901: 1-3). Alles was das Individuum für sich, seine Familie, für Gemeinde und Staat erarbeitete (auch Dienstleistungen und Handel), gehörte seiner Ansicht nach zum Wirtschaftlichen, während er im Arbeiten für »höhere Zwecke« (erwähnt werden Turnen, Spazierengehen, Gesundheitspflege - man könnte aristotelisch von intrinsischer Motivation sprechen) keine wirtschaftliche Tätigkeit sah. Demgegenüber vertrat Mises die Meinung, dass »jedes bewußte Verhalten« Wirtschaften sei:

»In der Tat gelingt es auch unschwer, in jedem denkbaren menschlichen - bewussten Verhalten die Grundkategorien der Katallaktik [Lehre von den Tauschbeziehungen, d. Verf.] (Wert, Gut, Tausch, Preis, Kosten) nachzuweisen. [...] Auch der Sprachgebrauch des Alltags gibt uns dafür genügend Beweise. Man beachte zum Beispiel wie außerhalb jenen Bereiches, den man im Alltag als Wirtschaft zu bezeichnen pflegt, Ausdrücke und Wendungen gebraucht werden, in denen von diesen Kategorien die Rede ist« (Mises 1933: 138).

Die Ökonomie sei die Wissenschaft, die sich mit dem »Vorziehen«, d. h. den Wahlakten zwischen sich bietenden Möglichkeiten beschäftige.

Das Knappheitsparadigma schlug sich im populärsten Handbuch zur Volkswirtschaftslehre des 20. Jahrhunderts erst verzögert nieder. 1948 erstmals erschienen, hatte »Economics« von Paul A. Samuelson die Ökonomie zunächst makroökonomisch vom Antagonismus zwischen der Depression und dem Boom, zwischen dem Aufschwung und der Krise her aufgerollt und die Aufgabe der modernen Nationalökonomie darin gesehen, die »Schwankungen des Volkseinkommens zu beschreiben, zu analysieren, zu erklären und ihre Wechselwirkungen zu bestimmen« (Samuelson 1948: 4-5). In der 5. Auflage von 1961 plädierte der blaue »Samuelson« dann dafür, »zum Zweck einer allgemeinen Einführung» die Knappheitsrelation als Definition der Ökonomie zu verwenden, woran bis heute festgehalten wurde:

»Die Volkswirtschaftslehre behandelt und prüft die Gesichtspunkte, nach denen sich der einzelne und die Gesellschaft im Zeitablauf entscheiden, die stets >knappen< Produktionsmittel - sei es mit oder ohne Verwendung von Geld - zur Güterproduktion heranzu- 
ziehen, und wie das Produktionsergebnis auf den heutigen und künftigen Konsum der einzelnen Individuen und der Gesellschaftsgruppen aufgeteilt wird « (Samuelson 1961: 20, Samuelson/Nordhaus 2005: 20-21).

\section{Expansion und Rückzug}

Der Verdienst (oder die Anmaßung), die Expansion der ökonomischen Methode explizit zum empirischen Programm der ökonomischen Wissenschaften erhoben zu haben, kommt dem amerikanischen Ökonomen Gary S. Becker zu. Becker hatte sich seit den 1950er Jahren mit Diskriminierung und "Human Capital« (insbesondere Bildung) beschäftigt (Becker 1964), als er Mitte der 1970er Jahre das Konzept der Ökonomie als Wissenschaft der Knappheitsrelationen zur Erklärung für jegliches menschliches Tun und Handeln erklärte (und keineswegs nur das bewusste wie noch bei Ludwig Mises):

»In der Tat bin ich zur Auffassung gekommen, dass der ökonomische Ansatz so umfassend ist, dass er auf alles menschliche Verhalten anwendbar ist, das monetär messbar ist oder unterstellte >Schatten -Preise hat, seien es wiederkehrende oder seltene Entscheidungen, seien es wichtige oder nebensächliche Entscheidungen, handle es sich um emotionale oder nüchterne Ziele, reiche oder arme Menschen, Männer oder Frauen, Erwachsene oder Kinder, kluge oder dumme Menschen, Patienten oder Therapeuten, Geschäftleute oder Politiker, Lehrer oder Schüler« (Becker 1976: 2).

Diesen Denkstil popularisierte er zwischen 1985 und 2004 auch als Kolumnist von Business Week (Becker/Becker 1997), nobilitiert wurde er 1992 durch die Verleihung des Nobelpreises für Ökonomie - für Beckers Werk, das »has extended the sphere of economical analyses to new areas of human behaviour« (Nobelprize.org).

Es gib seither keine Phänomene mehr, die nicht als Knappheitsrelation denkbar wären: Seien es wissenschaftliche Argumente (eine gesteigerte Nachfrage von Interessengruppen oder Auftraggebern führt gemäß Becker dazu, dass solche Argumente verstärkt angeboten werden), das Heiraten (ein Mensch heiratet, wie Becker empirisch nachgewiesen hat, wenn der erwartete Nutzen den Nutzen des Alleinbleibens oder der weiteren Suche nach einem passenden Partner übersteigt), oder Organtransplantationen (für die Becker die Zulassung von monetären Anreizen, d. h. die Legalisierung des Handels fordert, um das Angebot transplantierbarer Organe zu steigern). Die Ökonomie expandierte und erklärte sich zu einer allgemeinen Gesellschafts- und Handlungstheorie - 1990 durch das Diktum des Ökonomen Bruno S. Frey »Ökonomie ist Sozialwissenschaft« auf den Punkt gebracht (Frey 1990).

Wie halten es denn die Geisteswissenschaften mit den expandierenden Wissensbeständen der Ökonomie? Die Geschichtswissenschaften haben immer wieder auf ökonomisches Wissen zurückgegriffen, und zwar als Explanans, indem sie Theorien, Konzepte, Werkzeuge und Daten der Ökonomie für die historische Interpretation und Analyse verwendet haben. Klassischerweise waren dies Wis- 
sensbestände aus der Makroökonomie, wie beispielsweise die Konjunkturzyklen und Krisen, die in der neueren Sozialgeschichte seit den 1960er Jahren als weitreichendes Erklärungsmuster dienten und hinsichtlich der Auswirkungen auf die soziale Schichtung, das politischen Denken, die sozialen Bewegungen, die Mentalitäten, die Mode, die Musik, die Sexualität, den Gebrauch der Rauschmittel, sprich: alle denkbaren historischen Phänomene untersucht wurden. Die Konjunkturzyklen und Krisen bildeten auch ein vielfach verwendetes Gerüst für historische Narrative, beispielsweise für Eric Hobsbawms Synthese des 20. Jahrhunderts »Zeitalter der Extreme« (Hobsbawm 1994). Die Einteilung in drei Perioden (Katastrophenzeitalter, Goldenes Zeitalter und Erdrutsch) wird durch zwei Krisen- und eine Boomphase strukturiert.

Demgegenüber haben die Geschichtswissenschaften die ökonomischen Wissensbestände bislang noch kaum als Explanandum, d. h. als potentielle Forschungsobjekte betrachtet, die einer historischen Analyse unterworfen werden könnten. Das ist erstaunlich und erklärungsbedürftig, weil jene neueren Tendenzen in der Geschichtswissenschaft, die sich seit den 1980er Jahren zunehmend als historische Kulturwissenschaft verstehen, die Historizität jeglicher Diskurse, Praktiken und Artefakte betonen. Kulturhistoriker entwickelten zum Denken in (Schatten)Preisen, dem Kalkulieren von Kosten und dem Messen von Werten keine eigenen Forschungsfragen, solches ging in den letzten zwei Jahrzehnten inmitten von Körpern, Geschlechterdifferenzen, naturwissenschaftlichen Labors, Medien, Bildern, postkolonialen Identitäten, Sprachbildern und Metaphern, Kulturtechniken und Naturgesetzen unter. Die Omnipräsenz ökonomischer Referenzen in Sprache und Alltag seit Ronald Reagan, Margaret Thatcher und Gary S. Becker korrelierte mit einer Absenz des Ökonomischen in der Kulturanalyse. Diese Abstinenz der Geisteswissenschaften gegenüber ökonomischen Fragen korrelierte zeitlich mit einem Rückzug der an die Ökonomie angegliederten Wirtschaftsgeschichte aus einem allgemeinen geschichtswissenschaftlichen Diskurs. Als im Jahr 2008 die Bilder von Analysten, die mit Kartonschachteln auf die Strassen Manhattans strömten, in den Feuilletons eine neue Konjunktur des Redens über Hedgefonds, Too Big to Fail und Subprime Papiere auslöste, hatten Kulturhistoriker wenig zu sagen darüber, wie die Welt der Analysten, der Unternehmensberater und der Ratingagenturen entstanden war und auf welchen Kulturtechniken, Denkstilen und Assoziationen zwischen Akteuren, Zeichen und Artefakten sie beruhte, obwohl sie dies doch bei anderen Wissensgebieten wie den Life Sciences schon lange getan hatten. Und vor allem hatte niemand eine Erklärung dafür, warum die Ökonomie so lange unbeobachtet, außerhalb der Wahrnehmung und Kontrolle der Wissenschaft, der Politik und des Rechts geblieben war. Kulturwissenschaftler überließen das Feld den Ökonomen, den Marxisten oder auch den apokalyptischen Deutungen eines Peter Sloterdijks (»Wir leben in einer Frivolitätsepoche« 2008). 


\section{Exkursionen als Einmischung}

Die Geisteswissenschaften sollten auf die ökonomischen Expansionen mit eigenen kulturwissenschaftlichen Exkursionen in die von Ökonomen erschlossenen Terrains antworten, so wie die Ethnologen einst den Kolonialisten dicht auf den Fersen in die traurigen Tropen gefolgt sind. Doch wohin soll die Forschungsreise gehen? Und welche Fragen könnten dabei hilfreich sein?

Erstens sollte die kulturwissenschaftliche Exkursion im eigenen Haus beginnen. Kulturwissenschaftler sollten sich genau mit dem beschäftigen, mit dem sie sich bislang auch beschäftigt haben, d. h. mit Literatur, Kunst, Film, Körpern, Medien, Politik etc. Doch sollten sie dabei so vorgehen, wie der Journalist, der den Übersetzer nicht im Feuilleton, sondern in der Rubrik Geld interviewt, und damit beginnen, andere Fragen als üblich zu entwickeln (Hutter 2010). Beispielsweise läge es nahe, über neue Fragen zur Denkfigur des »Wertes« nachzudenken. Die Beschränkung der Kunst- und Literaturwissenschaften auf die ästhetischen Werte von Werken führte zu einem eingeschränkten Blick auf die Produktion, Zirkulation und Rezeption von Kunst, Literatur und Film und entkoppelte sie gleichsam künstlich von der Welt der Arbeitsverhältnisse, der Banken, Börsen und der Spekulation, mit der sie verhängt ist. Beim eingangs erwähnten Foster Wallace- und Analysten-Übersetzer aus Basel auf der Geldseite der Süddeutschen Zeitung laufen Hochwertigkeit (als ästhetisches Kriterium von Literatur) und Billiglohn (als Marktpreis von Übersetzungsarbeit) zusammen. Verschiedene Wertkategorien vermischen sich, indem der mit Wertschätzung und Preisen überhäufte Übersetzer sein finanzielles Manko durch Nachtarbeit »subventioniert« und die »Baukasten-Prosa« der Analysten übersetzt. Damit eröffnet sich ein Analysefeld, das sich in die Domäne der ökonomischen Theorie einmischt und zur Frage vordringen kann, inwiefern die Modelle der Wirtschaftswissenschaften den mannigfaltigen Wertphänomenen überhaupt Rechnung zu tragen vermögen.

Zweitens sollte der Existenz einflussreicher Theorieangebote der Ökonomie mit kulturwissenschaftlicher Empirie begegnet werden. Die Expansion ökonomischer Theorien wird gerade auch von Seiten der Geisteswissenschaften als Ökonomisierung des Sozialen und Kulturellen seit langem kritisiert. Doch zielte diese Kritik an der Ökonomie vorbei, die genau solches im Sinn gehabt hatte. Die Entwicklung der ökonomischen Theorien (in den ökonomischen Fakultäten in die bis zur Bedeutungslosigkeit degradierte Dogmengeschichte ausgelagert oder überhaupt längst abgeschafft) wäre ein wissenschaftliches Untersuchungsobjekt, dem die Kulturwissenschaften mit den durch die Science Studies entwickelten Methoden der Verfremdung der eigenen Kultur begegnen könnten (Latour 1987). Die Theorie der Knappheit, die damit eng verknüpfte Theorie der Opportunitätskosten, des Humankapitals oder das Prisoner's Dilemma der Spieltheorie (um nur einige Beispiele zu nennen) sind in spezifischen kulturellen Kontexten entstanden, haben sich im Lauf der Zeit verändert und sind in neuen Konstellationen gesellschaftlich einflussreich geworden. Sie sind mindestens so voraussetzungsund folgenreich wie etwa Konzepte der Objektivität in der Naturwissenschaft 
und müssten deshalb genau so gründlich mit Blick auf ihre Forschungssubjekte und Forschungsobjekte untersucht werden, wie dies Lorraine Daston und Peter Galison in ihrer wissenschaftshistorischen Analyse des Objektivitätsbegriffs getan haben (Daston 2007). Die Theorie der Knappheit ist von Michel Foucault in seinen Vorlesungen zur Geschichte der Biopolitik im Jahr 1978 und 1979 aufgegriffen worden, nachdem Gary S. Beckers programmatische Monographie zum ökonomischen Ansatz zwei Jahre zuvor in Chicago erschienen war (Foucault 2004: 309-314). Foucault hatte in der Einführung des Knappheitsparadigmas einen der Gründungsakte der neoliberalen Wirtschaftslehre gesehen und betont, dass der Homo Oekonomicus von Adam Smith und Karl Marx durch Gary S. Beckers und Lionel Robbins Knappheitsparadigma vollständig verändert wurde. Die Analyse von Arbeit war nun nicht mehr auf Tauschverhältnisse unter den Regeln von Angebot und Nachfrage ausgerichtet, sondern fokussierte auf die Bildung von Humankapital. Damit wurde ökonomisches Wissen anschlussfähig für neue Wissensbestände, beispielsweise die Genetik, die sich mit der Wahrscheinlichkeit zukünftiger Krankheiten aufgrund erworbener Anlagen beschäftigt und in Vereinigung mit Humankapital-Kalkulationen ein neues Feld von Wissen ermöglicht, das mit den bislang denkbaren Vorstellungen über die Lebensgestaltung des Individuums fundamental bricht. Michel Foucault drang damit ins Zentrum seines Interessengebietes der Biopolitik vor, verließ aber gleichzeitig die Analyse des ökonomischen Denkens vorschnell wieder, womit er (wie so oft) ein brachliegendes, fruchtbares intellektuelles Feld zurückließ. Es stellt sich nämlich die Frage, worin der epistemische Bruch des Knappheitsparadigmas und seine sozialen und kulturellen Auswirkungen eigentlich bestehen, wie er möglich wurde und welche neuen Konstellationen von Wissen neben der Genetik damit einhergehen.

Drittens stellt sich die Frage, ob die im Zusammenhang mit der Kritik an einer »Ökonomisierung« vorgebrachte These der Ausdehnung des ökonomischen Denkens auf »neue Bereiche« wirklich haltbar ist. Hat die Wahrnehmung des ökonomischen Denkens als gesellschaftlich expandierende und moralisch erodierende Macht das ökonomische Denken und Handeln nicht schon seit viel Längerem begleitet? Der Ökonomen Albert O. Hirschmann hat in seinen historischen Studien zur kulturellen Wahrnehmung des Kapitalismus (heutige Ökonomen würden von Preismechanismus sprechen) gezeigt, dass die Rede vom Zerfall moralischer Werte den Diskurs über die Marktmechanismen seit dem 18. Jahrhundert begleitet hat (Hirschman 1977). Es wäre für die historisch sensibilisierten Kulturwissenschaften (im Gegensatz zur komplett ahistorischen Ökonomie) vielleicht fruchtbar, der Frage nach der Geschichte von Tabus und Tabubrüchen im Zusammenhang mit dem Denken in Preisrelationen und Knappheitsparadigmas nachzugehen. Wann, in welchen Konstellationen und bei welchen Themen wurde das Denken in Werten, Geldmaßstäben und Marktmechanismen möglich, und wann und bei welchen Themen wurde dies kritisiert, gesellschaftlich sanktioniert und verhindert? Welche kulturelle Bedeutung hat es, dass ausgerechnet die mit den menschlichen Leidenschaften am innigsten assoziierten kulturellen Praktiken wie das Glücksspiel, die Prostitution und die berauschenden Stoffe bis heute 
nicht den Preismechanismen unterstellt werden? Und worin genau liegt die eigentliche Provokation eines Gary S. Beckers, wenn er die Liebe, den Tausch menschlicher Organe und zwischenmenschliche Geschlechts- und Rassenvorurteile mit dem Blick des Knappheitstheoretikers betrachtet?

Vielleicht liegt in der Abstinenz der Kulturwissenschaften, ökonomische Fragen in ihre Forschungsprojekte zu integrieren, ein Rest der Sehnsucht nach einer paradiesischen Vorstellung einer reinen Kultur jenseits der verzerrenden und zersetzenden Effekten der Welt der Wirtschaft.

\section{Literatur}

Becker, Gary S. (1964): Human Capital. A Theoretical and Empirical Analysis, with Special Reference to Education. National Bureau of Economic Research, New York, NY/London: Columbia UP.

Becker, Gary S. (1982): Der ökonomische Ansatz zur Erklärung menschlichen Verhaltens [1976], übers. v. Monika/Viktor Vanberg, Tübingen: Mohr.

Becker, Gary Stanley/Nashat, Guity (1998): Die Ökonomik des Alltags. Vom Baseball über Gleichstellung zur Einwanderung: Was unser Leben wirklich bestimmt [1997], übers. v. Gerhard Engel, Tübingen: Mohr.

Daston, Lorraine/Galison, Peter (2007): Objectivity, New York, NY: Zone Books.

Foucault, Michel (2004): Geschichte der Gouvernementalität II. Die Geburt der Biopolitik. Vorlesung am Collège de France, 1978-1979, hg. v. Michel Sennelart, Frankfurt a.M.: Suhrkamp.

Frey, Bruno S. (1990): Ökonomie ist Sozialwissenschaft. Die Anwendung der Ökonomie auf neue Gebiete, München: Franz Vahlen.

Grimm, Jakob und Wilhelm (1873): »Knapp«. In: Deutsches Wörterbuch, Bd. 5, Stuttgart: Hirzel, Sp. 1338-1348.

Hirschman, Albert O. (1977): Leidenschaften und Interessen. Politische Begründungen des Kapitalismus vor seinem Sieg [1977], Frankfurt a.M.: Suhrkamp.

Hirschman, Albert O. (1982): »Rival Interpretations of Market Society. Civilizing, Destructive, or Feeble?«. In: Journal of Economic Literature 20, H. 4, 1463-1484.

Hobsbawm, Eric (2009): Das Zeitalter der Extreme. Weltgeschichte des 20. Jahrhunderts [1994], übers. v. Yvonne Badal, 9. Aufl., München: dtv.

Hutter, Michael (2010): Wertwechselstrom. Texte zu Kunst und Wirtschaft, Hamburg: Philo.

Justi, Johann Heinrich Gottlob von (1969): System des Finanzwesens nach vernünftigen, aus dem Endzweck der bürgerlichen Gesellschaften und aus der Natur aller Quellen der Einkünfte des Staats hergeleiteten Grundsätze und Regeln abgehandelt [1766], Aalen: Scientia Verlag.

Latour, Bruno (1987): Science in Action: How to Follow Scientists and Engineers Through Society, Cambridge, MA: Harvard UP. 
Mises, Ludwig (1933): Grundprobleme der Nationalökonomie. Untersuchungen über Verfahren, Aufgaben und Inhalt der Wirtschafts- und Gesellschaftslehre, Jena: Gustav Fischer.

Mühlauer, Alexander/Wilhelm, Hannah (2010): Reden wir über Geld. Ulrich Blumenbach, http://sueddeutsche.de/geld/reden-wir-ueber-geld-blumenbachich-war-die-petersilie-auf-dem-salat-1.1006767, 19. Mai 2011.

N.N. (2008): »)Wir leben in einer Frivolitätsepoche<: Ein Gespräch mit dem Philosophen Peter Sloterdijk über die Finanzmarktkrise«. In: NZZ, 29. November 2008, http://www.nzz.ch/nachrichten/kultur/aktuell/wir_lebten_in_einer_frivolitaetsepoche_1.1324950.html, 17. Mai 2011.

Nobelprize.org: »The Prize in Economics 1992 - Press Release«, http:// nobelprize.org/nobel_prizes/economics/laureates/1992/press.html, 13. Mai 2011.

Robbins, Lionel (1952): An Essay on the Nature \& Siginificance of Economic Science [1932], London: Macmillan.

Samuelson, Paul A. (1952): Volkswirtschaftslehre. Eine einführende Analyse [1948], übers. v. Wirtschaftswiss. Institut der Gewerkschaften KölnBraunsfeld, Köln: Bund Verlag.

Samuelson, Paul A. (1964): Volkswirtschaftslehre. Eine Einführung [1961], übers. v. Wilhelm Hankel, Köln: Bund Verlag.

Samuelson, Paul A./Nordhaus, William (2005): Volkswirtschaftslehre. Das internationale Standardwerk der Makro- und Mikroökonomie, übers. v. Regina Berger, Landsberg am Lech: Redline.

Schmoller, Gustav (1901): Grundriss der Allgemeinen Volkswirtschaftslehre, Leipzig: Duncker \& Humblot.

Smith, Adam (2005): Der Wohlstand der Nationen. Eine Untersuchung seiner Natur und seiner Ursachen, 5. Aufl. [1789], München: dtv. 
Brought to you by | Universitaetsbibliothek Basel

Authenticated Download Date | 10/17/17 9:36 AM 